\title{
The Evolution of Proteomics from 2010 to 2020
}

\author{
Razan Kamal \\ Molecular Sciences, Macquarie University, Sydney 2150, Australia \\ *Corresponding author: Razan Kamal; razan-ghazi-a.kamal@students.mq.edu.au
}

Received 16 February 2022;

Accepted 03 March 2021;

Published 07 March 2022

\begin{abstract}
This paper presents a brief introduction to proteomics and defining the concept of proteomics. After that, listing some important techniques that used in proteomics from 2010 to 2020. It explores proteomics' techniques advantages and limitations, and how it has advanced in this past decade. Finally, this paper outlines the proteomics' evolution and its methods, techniques and modifications made in that period. This paper aim is to analyse and identify the changes and transitions that proteomics has experienced from 2010 to 2020.
\end{abstract}

Kevwords: Proteomics, last decade, Top-down proteomics, Middle-down proteomics, Mass spectrometry, MALDI-TOF MS

\section{Introduction}

New and improving technologies continuously impact biological research in different ways. Proteomics is the systematic study of proteins, their properties, and their role in the function of biological systems that help in many areas such as health and infections ${ }^{[1]}$. The approach of proteomics technologies worldwide helps in the detection and quantitation of proteins which can create new chances and challenges to understand diseases. Moreover, advance bioinformatics connecting with high-throughput proteomics technologies that used to recognise molecular marks of illnesses based on protein pathways ${ }^{[2]}$.

The applications of proteomics are diverse, and they are always coupled with other techniques such as two-dimensional gel electrophoresis and shotgun proteomics ${ }^{[3]}$. Rapid technological developments have also positively impacted resolution, mass accuracy, and sensitivity in protein analysis.

In 1907, physicist Joseph John Thomson began using mass spectrometry to visualising ions and analyse proteins. This technique was essentially used for separating and classifying atomic ions by mass ${ }^{[4]}$. Mass spectrometry (MS) has created significant improvements for large-scale studies in protein analysis. This review paper was studying and summarising the adaptation and changes in technology technic in the last decade which was from 2010 to 2020 .

\section{Techniques}

\section{Shotgun proteomics}

Shotgun proteomics is defined as 'multidimensional separating of protein mixtures produced from the specific protease such as trypsin and then analysed by MS, ${ }^{[5]}$. An important method for recognising and quantifying proteins is using a mixture of liquid chromatography combined with mass spectrometry ${ }^{[6,7]}$. In addition, this has an important role in MS-based proteome analysis, which has made charting of protein-to-protein interaction systems and quantitative reviews with modifications of protein posttranslational ${ }^{[7]}$. From 2010 to 2020 shotgun proteomics was used in many fields. For example, in in 2009 research was focusing on neurological diseases, by using shotgun proteomics-based tandem mass spectrometry that helps in the identification of proteins in a compound mixture ${ }^{[8]}$. Moreover, in 2014, research on microbial groups concentrating on specimen preparation and prefractionation on one cell type used shotgun proteomics ${ }^{[5]}$. Further, in 2020, research teams at Macquarie University analysed and identified key proteins in the leaves of rice plants by using label-free shotgun proteomics ${ }^{[9]}$.

Shotgun proteomics is one of the significant techniques used to recompense the limitations of Two-dimensional gel electrophoresis 2-DE. This is because the shotgun protein plays a vital role in digesting total protein as a mixture to separate peptides by using single- or multidimensional chromatography. The advantages are sensitivity, dynamic range, molecular weight, and hydrophobicity ${ }^{[6]}$.

Shotgun proteomics techniques have been used in increasingly wider-ranging applications. In 2006 Shotgun proteomics was involved in isobaric tagging of peptides to allow high-throughput protein analysis. Using iTRAQ reagents enabled concurrent identification and quantification of proteins with other specimens of prokaryotic and eukaryotic cells by using tandem mass spectrometry (MS). There were limitations, as it requires improvement of the total proteome coverage in obtaining a shotgun proteomics analysis ${ }^{[10]}$.

In 2020, shotgun proteomics was used jointly with MaxQuant software, which adds ion mobility to enhance proteome coverage, quantification accuracy, and dynamic range. There was significant difficulty as obtaining good computational reviews for 
large datasets with several LC-IMS-MS/MS runs being analysed together in one project ${ }^{[11]}$.

Other main applications were used in shotgun proteomics in 2013. 'Bottom-up' focuses on analysing peptides released from the protein throughout proteolysis. Protein identification is made by comparing the tandem mass spectra with theoretical tandem mass spectra that had been produced from silicon and separated in proetid database ${ }^{[10]}$. However, bottom-up has weaknesses such as loss of location of post-translational (PTMs), which can occur when proteins digested peptides ${ }^{[12]}$.

In addition, in 2014 research focused on 'top-down' proteomics' facts and perspectives. Top-down was first used to characterize intact proteins, specifically, undigested proteins, which measured more than $200 \mathrm{kDa}$. Other research has discovered more than 1,000 proteins by using multi-dimensional separations of multiple samples. However, there were some limitations with sensitivity and proteome coverage. Even with all of these difficulties, the top-down technique identifies as an essential complement and option to digestion-based approaches ${ }^{[13]}$.

Further, research merging the strengths of top-down and bottom-up, in 'middle-down' proteomics, was done in 2015. Middle-down proteomics is focusing on proteins that being digested in equal size when they are in a lysate. This has many advantages, such as higher mass accuracy and resolution ${ }^{[7]}$.

Due to the recent interest in top-down proteomics as MS technology has improved, there have been major improvements in many areas, such as improved efficiency in the separation of liquid chromatography (LC). Enhancements have also been made in data analysis software. Furthermore, in 2020 quantitative top-down MS was applied, which aimed to study the biological roles of protein proteoforms. There were drawbacks to using the top-down method, such as difficulty in detecting highly-charged proteins ${ }^{[12]}$.

To conclude that, research on shotgun proteomics indicates high growth in the scope of the proteomics field. Future research will focus on top-down quantitative techniques, which have been used to recognise biomarkers and in the study of bacterial infection in humans. Furthermore, improved identification of novel revolutionary proteases could help improve the field of bottom-up and middle-down proteomics ${ }^{[7,12]}$.

\section{Mass spectrometry}

One of the most important techniques used in proteomics is mass spectrometry. This method is used to identify and analyse chemical substances and unknown compounds by sorting volatile ions in electrical and magnetic fields due to mass-to-charge ratios ${ }^{[4]}$. Mass spectrometry is used in many fields such as pharmaceuticals, clinical research, and environmental studies. It is also used for discovering syntheses for medicines and their metabolites and endogenizing mixtures like peptides ${ }^{[14]}$.

Moreover, in 2003, tandem mass spectrometry was used in analysing metabolic disorders in newborns. This method was more sensible, specific, robust, and comprehensive than other old-style MS assays. Tandem MS was used as a detector to classify the weight of particles and their fragments ${ }^{[15]}$. There were limitations, however, such as low false-positive rates.

Research in 2012 on the technical development of liquid chromatography combined with tandem mass spectrometry (LCMS/MS) produced technology that was able to be used in most clinical laboratories around the globe, especially with its advantages of high accuracy and analytic multiplexing ability. Still, some significant disadvantages, such as selectivity regarding the appearance of 'isobaric' and lack of traceability, may affect the proficiency of testing ${ }^{[16]}$.

However, this year, there have been improvements in liquid chromatography-tandem mass spectrometry (LC-MS) regarding the qualitative and quantitative analysis. There are still weaknesses to identify and correct, such as isomeric syntheses, wrong mass formula and ionization inefficiency of the mixtures ${ }^{[17]}$. More 2020 research is focusing on gas chromatography-mass spectrometry GC-MS, to be used as alternative to Liquid chromatography-mass spectrometry (LC-MS), especially on pesticide residue determinations. It was noted that the GC-MS technique is more sensitive and specific ${ }^{[18]}$.

Another approach taken in 2015 was GC-Triple Quadrupole MS, which is electronic ionisation done by increasing ionic energy to achieve the highest level of precursor ions for the steroid hormones. This technique was used successfully with high accuracy and selectivity but was somewhat limited in detection ${ }^{[19]}$. In addition, in 2020 there was some development with respect to those reported in the past, and the results with regard to linearity and precision were acceptable ${ }^{[20]}$.

MALDI-IMS (MALDI imagining mass spectrometry) is defining as a matrix-assisted laser desorption ionization for imagining mass spectrometry ${ }^{[21]}$. However, from 2010 to 2015, was an important tool for molecular imaging of biological tests. MALDI-IMS has specific features for analysing tissue samples in a unique way. It can analyse protein modification, drugs and lipids. MALDI-IMS' advantages allow for checking the location of known and unknown compounds without requiring labelling, and in helping in biomarker identification and effectiveness. Limitations were low sensitivity of the mass range and molecular detection capability regarding MS/MS incapability ${ }^{[22,23]}$.

However, this year there is some development in the method for analysing in MALDI-IMS. was used in studying lipid disorders in fat tissues, and there were improvements in analytical performance $^{[24]}$.

Moreover, MALDI-TOF MS (time-of-flight mass spectrometry) has been used in many fields. And in 2010, was used in identifying microorganisms such as Archaea, viruses. And was used in microbiology labs as pathogenic identification in colony and blood culture. It was easy to use, fast and cost-effectiveness. However, there were some disadvantages such as recorded errors in identification process and capacity of MALDI TOF-MS in identifying cell lines was near to weak. Nevertheless, it is an economical choice on a widespread basis, but it needs to improve interpretation significantly ${ }^{[25]}$.

In 2020, MALDI-TOF MS was improved and used as identification of mosquitoes from New Caledonia, and the results of which showed species classification accurately with high sensibility and specificity ${ }^{[23]}$. It also applied to the rapid classification of microorganisms separate from clinical samples. It has many advantages, such as being inexpensive and quick, with high quality for identification ${ }^{[24]}$.

Finally, the facts indicate that research on MS is always improving. New, developing techniques make analysis simpler, cheaper, and more precise. Moreover, MS proteomics has generated high interest in the scientific community, and will be featured at the 8th Global Conference on Mass Spectrometry on September and at the 9th Global Mass Spectrometry in October $2020^{[26]}$.

All these improvements in MS instrument technology will lead to many new discoveries in protein identification and MS essential for every clinical laboratory. ${ }^{[16]}$. 


\section{Proteomics in Australia 2010-2020}

In the last decade, Australia has contributed significantly to the research and application of proteomics, which has been used in many fields such as agriculture, health, and food quality and safety.

In 2019 the Commonwealth Scientific and Industrial Research Organisation (CSIRO) conducted research focused on identifying proteins that led to improved health in Australia's livestock and plants. The research had conducted using mass spectrometry (MS) ${ }^{[27]}$.

Moreover, The Australasian Proteomics Society (APS) has concentrated on proteomics research with the intention of spreading its knowledge to students in the field ${ }^{[28]}$. Founded in 1994, the APS, a department within Macquarie University for Molecular Sciences, aims to be a market leader in proteomics expertise. In addition, Macquarie University's research has highly impacted Australian industry ${ }^{[29]}$, For example, enhancing crop yields and wheat support technique. In 2016 research was conducted on 'Quantitative proteomic analysis of two different rice varieties reveals' ${ }^{[30]}$. And in this year Macquarie University's published a research about crude protein effect in broiler chickens. The responses recognised as an outcome of this study was collected in the Australian Proteome Analysis Facility (APAF) ${ }^{[31]}$.

\section{Proteomics in Saudi Arabia 2010-2020}

In 2011 Saudi Arabia has a large improvement in clinical proteomics, advancing mass spectrometry and the Proteomics Society was established. However, advancing MS was used in many areas such as biotechnology, energy fields and pharmaceuticals ${ }^{[32]}$.

In 2020 proteomics approach was applied in medical diagnosis research by using highly developed techniques such as LC-MS/MS, SELDI-TOF/MS and MALDI-TOF/MS which assist in discovering new biomarkers, detecting diseases and observance patient care ${ }^{[33]}$.

In the same year, King Abdullah International Medical Research has published characterization of a new cell line in "protein and phosphoprotein profiling of KAIMRC1 comparison to MDA-MB-231 and MCF-7" by using mass spectrometry (MS). And this cell line can be applied for screening targets as anticancer drugs ${ }^{[34]}$.

\section{Conclusion}

This review's initial objectives were the classification and quantification of proteins. Few techniques and methods were available to perform the research for this paper. This review has studied changes of proteomics over the last decade in technology, methods, and techniques, in shotgun proteomics and pass spectrometry techniques.

Recently, increased funding for this emerging application has enabled the development of new methods and techniques to increase proteomics knowledge. The main difficulties that proteomics meets at this moment are in detecting highly charged proteins in top-down proteomics and classifying isomeric syntheses in liquid chromatography-tandem mass spectrometry. However, continued research is crucial in achieving better understanding and awareness of biological systems, in tandem with the development of methods and machines that assist the studying of proteins, peptides, and their functions. Finally, it is expected that further study will lead to advancements in methods and techniques that will continue have wide-reaching impact far beyond 2020 .

\section{Ethics approval and consent to participate}

This study included no participate and there was no need for either ethical approval or consent.

\section{List of abbreviations}

Mass spectrometry (MS)

Two-dimensional gel electrophoresis (2-DE)

Isobaric tags for relative and absolute quantitation (iTRAQ)

liquid chromatography-ion mobility spectrometry-mass

spectrometry (LC-IMS-MS)

Post translational modifications (PTMs)

liquid chromatography (LC)

Liquid Chromatography with tandem mass spectrometry (LC-MSMS)

liquid chromatography-tandem mass spectrometry (LC-MS)

gas chromatography-mass spectrometry (GC-MS)

MALDI mass spectrometry imaging (MALDI-MSI)

time-of-flight mass spectrometry (MALDI-TOF MS)

Matrix-Assisted Laser Desorption/Ionization Time-of-Flight Mass Spectrometry (MALDI-TOF MS)

Commonwealth Scientific and Industrial Research Organisation (CSIRO)

Australasian Proteomics Society (APS)

Australian Proteome Analysis Facility (APAF)

Surface-enhanced laser desorption/ionization time-of-flight mass spectrometry (SELDI-TOF-MS)

\section{Data Availability}

Data are available from the authors on request.

\section{Conflict of interest statement}

The author has declared no conflict of interest.

\section{Authors' contributions}

The work is done by the author of the manuscript.

\section{Acknowledgments}

The author would like to thank everyone who helped to publish this paper.

\section{References}

[1] S.D. Patterson, R.H. Aebersold, Proteomics: the first decade and beyond, Nat Genet 33 Suppl (2003) 311-23.

[2] W.C. Cho, Proteomics technologies and challenges, Genomics Proteomics Bioinformatics 5(2) (2007) 77-85.

[3] J.R. Coorssen, Brenner's encyclopedia of genetics, 2nd ed., Elsevier, University of Western Sydney, Campbelltown, NSW, Australia, 2013

[4] L. Brown, J.H. Beynon, Mass spectrometry, Encyclopædia Britannica (2020).

[5] S. Matallana-Surget, B. Leroy, R. Wattiez, Shotgun proteomics: concept, key points and data mining, Expert Rev Proteomic 7(1) (2010) 5-7.

[6] K. Kubota, T. Kosaka, K. Ichikawa, Shotgun protein analysis by liquid chromatography-tandem mass spectrometry, Methods Mol Biol 519 (2009) 483-94.

[7] L. Tsiatsiani, A.J. Heck, Proteomics beyond trypsin, FEBS J 282(14) (2015) 2612-26. 
[8] L. Liao, D.B. McClatchy, J.R. Yates, Shotgun proteomics in neuroscience, Neuron 63(1) (2009) 12-26.

[9] S. Hamzelou, D. Pascovici, K.S. Kamath, A. Amirkhani, M. McKay, M. Mirzaei, B.J. Atwell, P.A. Haynes, Proteomic Responses to Drought Vary Widely Among Eight Diverse Genotypes of Rice (Oryza sativa), Int J Mol Sci 21(1) (2020).

[10] K. Aggarwal, L.H. Choe, K.H. Lee, Shotgun proteomics using the iTRAQ isobaric tags, Brief Funct Genomic Proteomic 5(2) (2006) 112-20.

[11] N. Prianichnikov, H. Koch, S. Koch, M. Lubeck, R. Heilig, S. Brehmer, R. Fischer, J. Cox, MaxQuant Software for Ion Mobility Enhanced Shotgun Proteomics, Mol Cell Proteomics 19(6) (2020) 10581069.

[12] K.A. Cupp-Sutton, S. Wu, High-throughput quantitative top-down proteomics, Mol Omics 16(2) (2020) 91-99.

[13] A.D. Catherman, O.S. Skinner, N.L. Kelleher, Top Down proteomics: facts and perspectives, Biochem Biophys Res Commun 445(4) (2014) 683-93.

[14] J.F. Xiao, B. Zhou, H.W. Ressom, Metabolite identification and quantitation in LC-MS/MS-based metabolomics, Trends Analyt Chem 32 (2012) 1-14.

[15] D.H. Chace, T.A. Kalas, E.W. Naylor, Use of tandem mass spectrometry for multianalyte screening of dried blood specimens from newborns, Clin Chem 49(11) (2003) 1797-1817.

[16] C. Seger, Usage and limitations of liquid chromatography-tandem mass spectrometry (LCMS/MS) in clinical routine laboratories, Wien Med Wochenschr 162(21-22) (2012) 499-504.

[17] M. Beccaria, D. Cabooter, Current developments in LCMS for pharmaceutical analysis, Analyst 145(4) (2020) 1129-1157.

[18] Y. Pico, A.H. Alfarhan, D. Barcelo, How recent innovations in gas chromatography-mass spectrometry have improved pesticide residue determination: An alternative technique to be in your radar, TrAC Trends in Analytical Chemistry 122 (2020) 115720.

[19] S. Matysik, G. Schmitz, Determination of steroid hormones in human plasma by GC-triple quadrupole MS, Steroids 99 (2015) 151-154.

[20] P.M. Santos, L. Campo, L. Olgiati, E. Polledri, M. del Nogal Sánchez, S. Fustinoni, Development of a method to profile 2-4-ring polycyclic aromatic hydrocarbons in saliva samples from smokers and non-smokers by headspace-solid-phase microextraction-gas chromatography-triple quadrupole tandem mass spectrometry, Journal of Chromatography B (2020) 122273.

[21] L.A. McDonnell, R.M. Heeren, Imaging mass spectrometry, Mass spectrometry reviews 26(4) (2007) 606-643.

[22] M. Aichler, A. Walch, MALDI Imaging mass spectrometry: current frontiers and perspectives in pathology research and practice, Lab Invest 95(4) (2015) 422-431.

[23] N. Zaima, T. Hayasaka, N. Goto-Inoue, M. Setou, Matrix-assisted laser desorption/ionization imaging mass spectrometry, Int J Mol Sci 11(12) (2010) 5040-55.

[24] A. Fernández-Vega, E. Chicano-Gálvez, B. Prentice, D. Anderson, F. Priego-Capote, M. López-Bascón, M.
Calderón-Santiago, M. Avendaño, R. Guzmán-Ruiz, M. Tena-Sempere, Optimization of a MALDI-Imaging protocol for studying adipose tissue-associated disorders, Talanta (2020) 121184

[25] P. Seng, J.-M. Rolain, P.E. Fournier, B. La Scola, M. Drancourt, D. Raoult, MALDI-TOF-mass spectrometry applications in clinical microbiology, Future microbiology 5(11) (2010) 1733-1754.

[26] S. Conferences, OCM for Mass Spectrometry Series Conferences, (2020).

[27] C.S.a.I.R.O. (CSIRO), Protemics (2019).

[28] T.A.P.S. (APS), (2020).

[29] M. University, Australian Proteome Analysis Facility.

[30] Y. Wu, M. Mirzaei, D. Pascovici, J.M. Chick, B.J. Atwell, P.A. Haynes, Quantitative proteomic analysis of two different rice varieties reveals that drought tolerance is correlated with reduced abundance of photosynthetic machinery and increased abundance of ClpD1 protease, $\mathrm{J}$ Proteomics 143 (2016) 73-82.

[31] S. Greenhalgh, B.V. McInerney, L.R. McQuade, P.V. Chrystal, A. Khoddami, M.A. Zhuang, S.Y. Liu, P.H. Selle, Capping dietary starch: Protein ratios in moderately reduced crude protein, wheat-based diets showed promise but further reductions generated inferior growth performance in broiler chickens from 7 to 35 days post-hatch, Animal Nutrition (2020).

[32] A.A. Peer-Zada, M.H. Al-Qahtani, Advancing mass spectrometry-based clinical proteomics in Saudi Arabia, Saudi Med J 32(3) (2011) 225-235.

[33] R.A. Alharbi, Proteomics approach and techniques in identification of reliable biomarkers for diseases, Saudi journal of biological sciences 27(3) (2020) 968-974.

[34] B. Alghanem, R. Ali, A. Nehdi, H. Al Zahrani, A. Altolayyan, H. Shaibah, O. Baz, A. Alhallaj, J.J. Moresco, J.K. Diedrich, Proteomics Profiling of KAIMRC1 in Comparison to MDA-MB231 and MCF-7, International journal of molecular sciences 21(12) (2020) 4328.

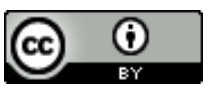

Open Access This article is licensed under a Creative Commons Attribution 4.0 International License, which permits use, sharing, adaptation, distribution and reproduction in any medium or format, as long as you give appropriate credit to the original author(s) and the source, provide a link to the Creative Commons license, and indicate if changes were made. The images or other third party material in this article are included in the article's Creative Commons license, unless indicated otherwise in a credit line to the material. If material is not included in the article's Creative Commons license and your intended use is not permitted by statutory regulation or exceeds the permitted use, you will need to obtain permission directly from the copyright holder. To view a copy of this license, visit https://creativecommons.org/licenses/by/4.0/.

(C) The Author(s) 2021 